\title{
Knee Joint Alignment in the Indigenous People of Sri Lanka
}

\author{
H. A. Amaratunga ${ }^{1}$, S. B. Adikari ${ }^{1}$, M. R. Ileperuma ${ }^{2}$, G. H. M. D. N. Chandrasekara ${ }^{2}$, \\ M. S. Chandrasekara ${ }^{1}$, H. J. Suraweera ${ }^{2}$
}

\begin{abstract}
Objective: The objective of this study was to measure the knee joint alignment of the indigenous people of Dambana using the goniometer and compare with a control group of Sinhalese individuals.

Materials and Methods: Hundred adult volunteers, from the population of "veddas" living in Dambana and 100 adult Sinhalese volunteers above the age of 25 were recruited for the study. Weight and height of the subjects were measured and the BMI calculated using the Quetelets index. The knee joint alignment was measured using the goniometer according to the method described by Kraus et al. (2005).

Results: Indigenous people included 46 females and 54 males with an age range of 26 to 75 years. The control sample consisted of 50 females and 50 males with an age range of 25 to 65 years. The BMI of indigenous people was 21.19 and that of the control population was 22.5. Indigenous people had a mean knee alignment angle of $182.4^{\circ}$ and the control sample had a mean of $180.9^{\circ}$. None of the over 50 years population had clinical evidence of osteoarthritis $(\mathrm{OA})$ in the indigenous group while in the control group, all females above 50 years had mild knee pain and 2 had clinically detectable OA.

Conclusions: Knee joint alignment in the indigenous population is closer to the normal range than in the control sample. Clinically detectable OA appears to be absent among the indigenous people.
\end{abstract}

Key words: knee joint alignment, knee osteoarthritis, indigenous people

\section{INTRODUCTION}

The alignment of the knee joint has generated much interest in the recent past due to its link with osteoarthritis (OA) (1). It is described as the angle formed by the femoral shaft and the tibial shaft at the knee. The exact factors that influence the alignment of the knee have not been elucidated to date. However, it is postulated that genetic factors, body mass index (BMI), laxity of the joint and the life style may play a role (2). A full length anteroposterior weight bearing limb radiograph is considered as the gold standard for measuring the alignment of the knee (3). Goniometry is an alternative and simpler method to physically measure the alignment and matches well with the gold standard measurement (4,5). Being a physical examination method it does not expose the patient to radiation and can be easily incorporated into the knee examination.

The indigenous population of Sri Lanka, are commonly known as the "veddas" live in a relatively closed community, with many inter marriages. They have a way of life which causes them to squat, climb trees, carry heavy weights and walk long distances. This type of life style leads to strain on the knee joints. Strain on the knee 
joints has been associated with development and progression of OA $(6,7)$. Due to this life style and the high fiber content of their food obesity is relatively uncommon in their population.

Therefore, the objective of this study was to measure the knee joint alignment of the indigenous people and compare with a group of urban Sinhalese individuals and to detect $\mathrm{OA}$ of the knee joint by clinical examination.

\section{MATERIALS AND METHODS}

Hundred adult volunteers above the age of 25 years, from the population of indigenous people living in Dambana were included in the study. The other group consisted of 100 adult Sinhalese volunteers from the University population above the age of 25 years.

In both groups weight and height were measured in kilograms and meters using standard measuring scales and the BMI was calculated using the Quetelets index (8). The knee joint alignment was measured using the goniometer according to the method described by Kraus et al. (5). Each subject was carefully questioned with regard to the symptoms of knee joint OA. Both knees were examined for the presence of swelling, deformity, tenderness and crepitus. A diagnosis of OA was made using the clinical criteria laid down by the American College of Rheumatology. The criteria being, knee pain with morning stiffness lasting 30 minutes or less and crepitus on motion (9).

\section{RESULTS}

The sample from the indigenous people consisted of 100 individuals who included 46 females and 54 males with an age range of 26 to 75 years. The control sample consisted of 50 females and 50 males with an age range of 25 to 63 years. Therefore 200 knees of the indigenous people and 200 knees of the control sample were measured. The mean BMI of the indigenous people was $21.19( \pm 3.95)$ and that of the control group was $23( \pm 3.2)$. The observed difference in the mean BMI was statistically significant. Indigenous people had a mean knee alignment angle of $182.4^{\circ}( \pm 3.45)$ and the Sinhalese sample had a mean of $180.9^{\circ}( \pm 4.7)$ (Figure 1$)$. However the difference observed in the means was not statistically significant. In the indigenous people highest number of knees were in the $181^{\circ}$ to $185^{\circ}$ range while in the control the highest number was seen in the range of 176 to $180^{\circ}$. The variance was lower than the Sinhalese in the indigenous population (Figure 2). None of the over 50 years population had clinical evidence of $\mathrm{OA}$ in the indigenous group while in the Sinhalese group, all females above 50 years (12) had mild knee pain and 2 had clinically detectable OA.

\section{DISCUSSION}

In studies done in the West the normal range for knee joint alignment angle as measured by the goniometer is considered as 182 to $184^{\circ}(5)$. It was observed that the knee angle range in the indigenous people was very close to this. However in the control population the range was several degrees less. The active life style of the indigenous people maybe resulted in an increased strength in the ligaments and muscle in the lower limbs, thereby maintaining the angle within the normal range. The lower BMI of the indigenous people is by reducing the load on the knees maybe maintaining the alignment closer to the normal range. The fact that the knee alignment angle is concentrated to a narrower range with low variance in the 


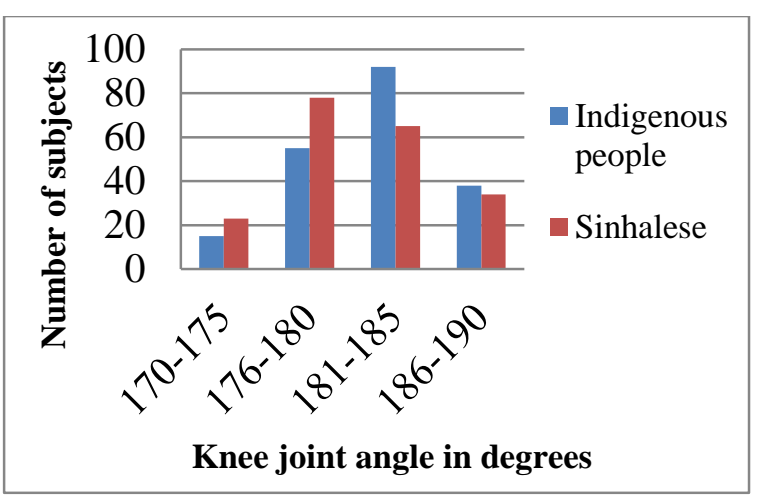

Figure 1: Distribution of the knee joint alignment in the indigenous and the Sinhalese population

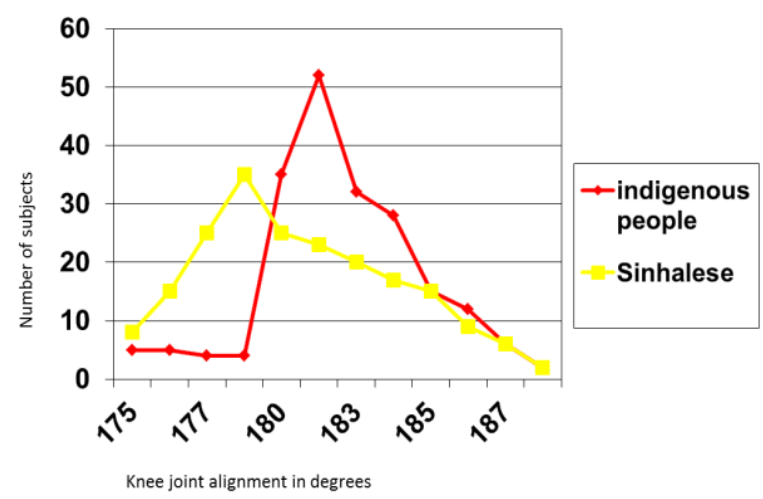

Figure 2: Variance in the knee joint alignment in the indigenous people and the Sinhalese group

indigenous people could be due to the closeness of the community and inter marriages leading to a restricted gene pool (10). Knee joint alignment in the indigenous population is closer to the normal range than in the control sample. However there is no significant difference between the mean knee alignment in the two groups. Clinically detectable OA appears to be absent among the indigenous people.

\section{REFERENCES}

1. Brouwer GM, van Tol AW, Bergink AP, Belo JN, Bernsen RM, Reijman M. Association between valgus and varus alignment and the development and progression of radiographic osteoarthritis of the knee. Arthritis Rheum, 2007; 56: 1204-11.
2. Hsu RW, Himeno S, Coventry MB, Chao EY. Normal axial alignment of the lower extremity and load-bearing distribution at the knee. Clin Orthop Relat Res. 1990; 255: 215227.

3. Moreland JR, Bassett LW, Hanker GJ. Radiographic analysis of the axial alignment of the lower extremity. J Bone Joint Surg Am, 1987; 69: 745-749.

4. Cibere J, Bellamy N, Thorne A, John M, Esdaile Kelly J, McGorm, Chalmers A, Huang, S, Peloso P, Shojania K, Singer J, Wong H, Kopec J. Reliability of the Knee Examination in Osteoarthritis Effect of Standardization. Arthritis Rheum. 2004; 50: 458-468.

5. Kraus VB, Vail TP, Worrell T, McDaniel GA. Comparative assessment of alignment angle of the knee by radiographic and physical examination methods. Arthritis Rheum. 2005: 52:1730-1735.

6. Schouten JS. An update on the relationship between occupational factors and osteoarthritis of the hip and knee. Curr Opin Rheumatol. 2002; 14: 89-92.

7. Maetzel A. Osteoarthritis of the hip and knee and mechanical occupational exposure-a systematic overview of the evidence. $J$ Rheumatol, 1997; 24: 1599-1607.

8. Garrow JS, Webster JD. Quetelets index as measurement of fatness. Int J Obes, 1985; 147.

9. Altman R, Asch E, Bloch D, Bole G, Borenstein D, Brandt K. Development of criteria for the classification and reporting of osteoarthritis. Diagnostic and Therapeutic Criteria Committee of the American Rheumatism Association. Arthritis Rheum. 1986; 29: 1039-1049.

10. Masatoshi N. Estimation of average heterozygosity and genetic distance from a small number of individuals. Genetics 1978: 2: 583-590.

\section{CORRESPONDENCE}

Dr. H. A. Amaratunga

Faculty of Medicine,

University of Peradeniya, Sri Lanka.

Email: himanipeiris@yahoo.com

Received: August 2017

Accepted: September 2017 